\title{
La bioprospección como un mecanismo de cooperación internacional para fortalecimiento de capacidades en ciencia y tecnología en Colombia
}

\author{
Oscar Duarte Torres \\ Doutorando em política científica e tecnológica. \\ Universidade Estadual de Campinas, São Paulo, Brasil. \\ E-mail:oscartorres@ige.unicamp.br
}

Lea Velho

Pós-Doutorado . Indiana University. Fundação de Amparo à Pesquisa do Estado de São Paulo. Pós-Doutorado. Cornell University. PósDoutorado. University of Edinburgh. Pós-Doutorado. Ohio State University. Doutorado em Política Científica e Tecnológica. University of Sussex, Sussex, Inglaterra.

E-mail:velho@ige.unicamp.br

\section{Resumen}

La bioprospección, definida como la exploración de la biodiversidad para fines comerciales, se ha convertido recientemente en una potencial estrategia mediante la cual los países ricos en biodiversidad pueden aumentar sus capacidades endógenas para realizar actividades de CyT, especialmente mediante la interacción con otros países de mayor desarrollo tecnológico. Es así, como este trabajo se concentra en analizar la mecánica de cooperación internacional de un país considerado megadiverso, Colombia, a través del análisis de coautoría de 342 artículos publicados en revistas indexadas en las redes ISI y SCOPUS, información que se complementa mediante entrevistas con 12 líderes de grupos colombianos. Los resultados evidencian que España es el país con mayor colaboración con Colombia; que la cooperación con países de América se sitúa en un tercer plano y ésta se intensifica cuando hay de por medio un país europeo; la escasa cooperación entre grupos colombianos origina una agenda de investigación dispersa y en temas muy variados sin orientación y prioridades estratégicas.

\section{Palabras clave}

Bioprospección. Colombia. Cooperación internacional. Coautoría. Bibliometría.

\author{
Bioprospecting an international cooperation \\ mechanism for strength the capabilities in \\ Science and Technology in Colombia
}

\begin{abstract}
Bioprospecting, defined as the exploration of biodiversitytargeted for commercial purposes, has recently become a potential strategy whereby biodiversity-rich countries can enhance their endogenous capacities to perform S\&T activities, especially by interacting with more technologically developed countries. Therefore, this work focuses on analyzing the mechanism of international cooperation of a megadiverse country, as Colombia, through analysis of 342 jointly authored articles published in journals indexed in ISI and SCOPUS, information that is complemented by 12 interviews of Colombian group leaders. Results thereof evidence that Spain is the country with the highest cooperation level with Colombia. Cooperation with American countries is considered as a third priority and is intensified with the participation of a European country. The poor cooperation among Colombian groups creates a disperse research agenda on widely varied topics lacking orientation and strategic priorities.
\end{abstract}

\section{Keywords}

Bioprospection. Colombia. International cooperation. Co-authored cooperation. Bibliometry. 


\section{INTRODUCCIÓN}

La bioprospección es definida como la búsqueda sistemática de componentes naturales y organismos completos de la biodiversidad con el fin de otorgarles un valor comercial para el desarrollo de productos (Castree, 2003; Laird, 2002; Laird y Wynberg, 2002; Feinsilver, 1996). La bioprospección genera productos que tienen relación con industrias como la farmacéutica, la biotecnológica, la de agro insumos y la de medicina botánica; entre otras; calculándose que en la actualidad una cuarta parte de los productos farmacéuticos son derivados de vegetales (Moran, et al, 2001). Es así, como se calcula que los mercados mundiales relacionados con la bioprospección, alcanzan sumas que varían entre US\$ 500 a US\$ 800 billones por año (Laird y ten Kate, 2002; Castree, 2003).

Para que los procesos de bioprospección se lleven a cabo se involucra una gama de interacciones que compromete desde el conocimiento tradicional de las comunidades locales e indígenas, pasando por el conocimiento científico y tecnológico de entes académicos e investigativos de los países del sur, hasta actividades comerciales de empresas multinacionales de países del norte. La interacción entre países del norte y del sur se propicia principalmente debido a que los primeros poseen recursos económicos y tecnológicos, mientras que la riqueza de los países del sur está representada principalmente en su biodiversidad y en el conocimiento local tradicional.

La heterogeneidad de intereses, recursos, capacidades, motivaciones y aspectos culturales de los diferentes actores que intervienen en esta interacción ha obligado al establecimiento de marcos mundiales, regionales y locales que regulen jurídicamente el acceso a los recursos genéticos y la distribución equitativa de los beneficios que se generen de dicha práctica. Ejemplo de esto es el llamado Convenio de Diversidad Biológica -CDB- (FAO, on line) y la Decisión Andina 391 (Comunidad Andina, Normatividad Andina, on line). Es en este punto en el cual autores como Brush (1999), Escobar (1999), Moran, et al (2001) y Boisvert y Caron (2002), cuestionan los reales beneficios de la bioprospección para los países del sur, argumentando que esta práctica se puede convertir en una forma de "bioimperialismo", mediante la cual los países del norte mediante esquemas de propiedad intelectual se apropian de recursos naturales.

Por tanto, la bioprospección es considerada un área interesante para análisis ya que en esta confluye una serie heterogénea de intereses y motivaciones (Brand y Görg, 2003) y en la cual es posible dinamizar el establecimiento de alianzas internacionales mediante las cuales se concreten mecanismos efectivos de cooperación que posibiliten a los países del sur la creación y fortalecimiento de capacidades internas para realizar acciones de ciencia y tecnología y de manera simultánea contribuyan para que los países del norte incrementen el conocimiento de la biodiversidad del sur y puedan obtener de ella productos comerciales que favorezcan aumentos en retribuciones económicas.

Un análisis de experiencias de cooperación internacional para realizar bioprospección (Georghiou, 1998; Henne y Fakir, 1999; Velho, 2001; Laird y Wynberg, 2002; Velho, 2004; Katsouyanni, 2008); indica que los principales intereses y motivaciones de los países ricos en biodiversidad están relacionados con el acceso y fortalecimiento a distintas clases de conocimientos y capacidades; lo cual está representado en la obtención de reconocimiento a nivel mundial del centro de investigación participante; el fortalecimiento de la infraestructura investigativa (equipos, laboratorios); aumento de oportunidades de entrenamiento del recurso humano mediante el acceso a técnicas especializadas; aumento en posibilidades para financiación de proyectos con recursos de fuentes internacionales; aumento en prestigio y visibilidad de investigadores y grupos de investigación a través de publicaciones compartidas; y en algunos pocos casos beneficios económicos a través del pago de regalías por la comercialización de productos. Las principales motivaciones de los países del norte se pueden dividir en dos vertientes que pueden estar interrelacionadas: la académica y la empresarial; en la académica, el principal interés es la posibilidad de acceder a la biodiversidad propia de agroecosistemas 
de zonas tropicales; desde la visión empresarial los intereses están motivados hacia el aumento de las ventas de productos con valor agregado provenientes de recursos primarios de la biodiversidad (Artuso, 2002); la diversificación de productos de base que suministren a la empresa una ventaja competitiva y la posibilidad de obtener derechos de propiedad intelectual sobre los productos obtenidos.

Las experiencias más analizadas y discutidas en las cuales un país biodiverso realiza contratos con empresas multinacionales para la exploración del potencial de sus recursos biológicos y genéticos se encuentran en Sur África (Instituto Nacional de Botánica de Sur África con empresa estadounidense Ball y la del Consejo de Investigaciones Científicas de este país con la empresa Pfizer) y Costa Rica (INBio, Instituto Nacional de Biodiversidad de Costa Rica con la empresa Merck). Un análisis de estas experiencias denota que el país del sur obtuvo beneficios relacionados con aportes importantes en tecnología, capacitación de recursos humanos y dotación de equipos de laboratorio (Cabrera, 2000; Laird y Wynberg, 2002; INBIO, on line); pero a pesar del relativo éxito mencionado algunos de los estudios realizados (Swiderska, 2001; Boisvert y Caron, 2002; Castree, 2003; Velho, 2004), llegan a conclusiones similares sobre la escasa construcción de competencias nacionales, la ausencia de una red de beneficiarios nacionales, la poca participación del sector empresarial nacional y la bajísima participación y beneficio de las comunidades locales.

Con relación a Colombia, al realizar un análisis de las experiencias documentadas de bioprospección (Melgarejo, et al 2002; Roa-Atkinson, 2004; Duarte y Velho, 2008), se detecta que esta práctica se ha abordado desde una óptica muy amplia en la cual se incluyen trabajos relacionados con toda la diversidad biológica del país, que un gran porcentaje de las actividades son desarrolladas por grupos científicos y tecnológicos con escasa participación de las empresas y las interacciones internacionales están representadas en su mayor parte por la relación entre grupos científicos y académicos de Colombia con grupos similares de otros países.
Es así, como este trabajo se concentra en analizar la mecánica de cooperación internacional a nivel de grupos de investigación científica y tecnológica de Colombia que trabajan en bioprospección; para lo cual se recurre al análisis de coautoría. Análisis que ha sido usado por varios autores ya que el número de publicaciones internacionales en coautoría es considerado como un indicador básico de cooperación científica internacional (Glanzel et. al, 1999). Las principales razones por las cuales se ha usado este indicador están relacionadas con (i) se espera que los resultados de la cooperación científica internacional se refleje en la literatura científica, la cual es una actividad mensurable (Glanzel et. al, 1999); (ii) refleja los flujos e intercambios del conocimiento asociado (Calero et. al, 2007); (iii) la proporción de artículos en coautoría dentro del Science Citation Index se ha venido incrementando de manera sostenida (Schmoch y Schubert, 2008; Katsouyanni, 2008); (iv) ha sido usado como indicador para detectar patrones del ambiente colaborativo y para encontrar tendencias por disciplina (Roa-Atkinson y Velho, 2005); (v) hay alguna base teórica y empírica que afirma que la coautoría puede tener relación con mayor calidad (Schmoch and Schubert, 2008); y (vi) sirve de base para monitorear el grado de excelencia científica y para establecer estándares confiables (Nicolini y Nozza, 2008).

Sin embargo, el análisis de coautoría internacional es un fenómeno calificado de complejo ya que está motivado por diferentes razones de las cuales aún no hay suficiente explicación (Schmoch and Schubert, 2008; Katsouyanni, 2008) y por lo tanto debe ser cuidadosamente limitado en sus análisis y alcances (Katz y Martin, 1997).

\section{METODOLOGÍA}

Con el fin de identificar la mecánica de cooperación internacional de los grupos colombianos que trabajan en bioprospección se tomó como base el análisis de coautoría; para lo cual se tuvo como referencia los 71 grupos colombianos de investigación científica y tecnológica que desarrollan 
actividades importantes en esta temática, grupos que fueron identificados a través de la plataforma ScienTI Colombia, administrada por el Instituto Colombiano para el Desarrollo de la Ciencia y la Tecnología -COLCIENCIAS- (COLCIENCIAS, web site), mediante el uso de las palabras clave "bioprospección", "prospección de la biodiversidad", "biocomercio", "bioactividad", "fitoquimica", "productos naturales", "conocimiento tradicional" y "recursos genéticos"; realizando la búsqueda a nivel de los campos de nombre del grupo, líneas de investigación, proyectos ejecutados o en ejecución y productos de los diferentes grupos de investigación. De cada uno de estos grupos se seleccionó la persona líder, cuyo nombre se consultó en la red ISI web of Science y en la red SCOPUS, con el fin de identificar los artículos de investigación en los cuales la persona líder aparece en la lista de autores (artículos hasta septiembre de 2008).

Se identificaron 342 artículos de 46 líderes (25 de los líderes no poseen artículos en las redes mencionadas), colectándose información relacionada con los siguientes aspectos: título del artículo, temática del artículo (con base en el abstract, palabras claves y título del artículo), año de publicación, título de la publicación, país editor de la publicación; instituciones y países participantes (acorde a la información institucional de cada autor mencionada en el respectivo artículo) y posición del autor colombiano en el orden de autores. Con base en esta información se realizó el análisis sobre las principales áreas temáticas, instituciones y países de cooperación internacional con Colombia en el área de bioprospección. Para analizar la relación cuantitativa de la participación de Colombia con los diferentes países, se usó la medida de Salton, definida como el número de publicaciones conjuntas entre dos países divido por la raíz cuadrada del número total de publicaciones (Glanzel et. al, 1999).

Con el fin de complementar la información colectada y profundizar en algunos de los aspectos detectados en el análisis bibliométrico, se realizaron entrevistas personales con 12 líderes de grupos colombianos que poseen publicaciones de las mencionadas anteriormente; realizándose una entrevista semiestructurada en la cual se indagó aspectos relacionados con las principales temáticas de cooperación, intereses y motivaciones para realizar procesos de cooperación con grupos de otros países, principales experiencias obtenidas y beneficios y responsabilidades de los grupos participantes.

\section{RESULTADOS Y DISCUSIÓN}

\section{Período de publicación de los artículos.}

Los 342 artículos identificados fueron publicados durante el período 1977 a 2008, de los cuales el $68 \%$ se publicaron en el período $2000-2008$ (tabla 1), observándose un aumento importante en el número de artículos publicados en revistas indexadas a medida que trascurrieron las décadas de los últimos 30 años, especialmente durante la última en la cual el promedio de artículos publicados por año se triplicó con relación a la década anterior. Esta tendencia incremental denota la importancia a nivel nacional e internacional de los temas relacionados con la bioprospección y el fortalecimiento endógeno de los grupos colombianos, los cuales han venido aumentado capacidades que les permite mayor visibilidad de sus actividades científicas y tecnológicas. Este aumento en capacidades de los grupos es el reflejo de políticas de $\mathrm{Cy} T$ implementadas por este país (COLCIENCIAS, 2008) con relación a estrategias orientadas al apoyo de estudios de posgrado en el

TABLA 1

Número de artículos publicados por líderes de grupos colombianos que trabajan en actividades de bioprospección, durante los últimos 30 años.

\begin{tabular}{l|c}
\hline Período & $\begin{array}{c}\text { Número artículos } \\
\text { publicados }\end{array}$ \\
\hline $2008-2000$ & 232 \\
\hline $1999-1990$ & 88 \\
\hline $1989-1980$ & 19 \\
\hline $1979-1977$ & 3 \\
\hline Total & 342 \\
\hline
\end{tabular}


exterior, fortalecimiento de programas nacionales de doctorado, apoyo y fomento a la consolidación de grupos de investigación, establecimiento de criterios internacionales para la categorización de grupos de investigación y apoyo a programas de movilidad internacional.

\section{Países e instituciones cooperantes}

De los 342 artículos mencionados anteriormente, 193 de ellos presentan relaciones interinstitucionales con 24 países (tabla 2).
Con base en la revisión de los artículos mencionados anteriormente y del análisis de las entrevistas personales realizadas a líderes de los grupos, se puede deducir lo siguiente con relación a la mecánica de cooperación internacional:

- De los 342 artículos, 43\% tienen como autores investigadores colombianos sin participación extranjera, lo cual ratifica que los grupos de este país han venido obteniendo fortalezas endógenas para adelantar trabajos y realizar publicaciones de buena calidad sin apoyo de cooperación internacional.

TABLA 2

Países con los cuales Colombia publica en el tema de bioprospección, distribuidos de acuerdo al número de artículos, tipo de institución y grado de cooperación (medida de Salton)

\begin{tabular}{|c|c|c|c|c|c|c|c|}
\hline \multirow[t]{2}{*}{ País } & \multirow{2}{*}{$\begin{array}{l}\mathrm{N}^{\circ} \\
\text { Art. }\end{array}$} & \multicolumn{5}{|c|}{ Instituciones } & \multirow{2}{*}{$\begin{array}{c}\text { Medida de } \\
\text { salton }\end{array}$} \\
\hline & & Univ. & CII & CNCT & Museos & Empr. & \\
\hline España & 59 & 18 & 1 & 1 & 0 & 2 & 3.89 \\
\hline Francia & 29 & 6 & 5 & 0 & 1 & 0 & 1.91 \\
\hline Alemania & 24 & 6 & 2 & 0 & 0 & 0 & 1.58 \\
\hline Japón & 20 & 1 & 1 & 0 & 0 & 0 & 1.32 \\
\hline E. Unidos & 19 & 7 & 2 & 1 & 0 & 0 & 1.25 \\
\hline Venezuela & 14 & 2 & 1 & 0 & 0 & 0 & 0.92 \\
\hline Argentina & 13 & 4 & 2 & 2 & 0 & 0 & 0.85 \\
\hline Chile & 12 & 5 & 0 & 0 & 0 & 0 & 0.79 \\
\hline Brasil & 10 & 8 & 2 & 0 & 0 & 0 & 0.66 \\
\hline Inglaterra & 7 & 9 & 0 & 0 & 0 & 1 & 0.46 \\
\hline Italia & 5 & 4 & 1 & 0 & 0 & 0 & 0.33 \\
\hline México & 4 & 2 & 1 & 1 & 0 & 0 & 0.26 \\
\hline Bolivia & 2 & 1 & 1 & 0 & 0 & 0 & 0.13 \\
\hline Suecia & 1 & 0 & 1 & 0 & 0 & 0 & 0.06 \\
\hline P. Rico & 1 & 1 & 0 & 0 & 0 & 0 & 0.06 \\
\hline Perú & 1 & 0 & 1 & 0 & 0 & 0 & 0.06 \\
\hline Canadá & 1 & 1 & 0 & 0 & 0 & 0 & 0.06 \\
\hline C. Rica & 1 & 1 & 0 & 0 & 0 & 0 & 0.06 \\
\hline Uruguay & 1 & 1 & 0 & 0 & 0 & 0 & 0.06 \\
\hline Suiza & 1 & 0 & 1 & 0 & 0 & 0 & 0.06 \\
\hline Bélgica & 1 & 1 & 1 & 0 & 0 & 0 & 0.06 \\
\hline China & 1 & 0 & 0 & 1 & 0 & 0 & 0.06 \\
\hline Sur África & 1 & 1 & 0 & 0 & 0 & 0 & 0.06 \\
\hline Indonesia & 1 & 0 & 1 & 0 & 0 & 0 & 0.06 \\
\hline TOTAL & 193 & 79 & 24 & 6 & 1 & 3 & \\
\hline
\end{tabular}

$\mathrm{N}^{\mathrm{o}}$ Art.= número de artículos; Univ.= Universidades; $\mathrm{CII}=$ Centros o Institutos de Investigación; $\mathrm{CNT}=$ Consejos Nacionales de Ciencia y Tecnología; Empr.= Empresas. 
En estos artículos hay participación de 35 instituciones colombianas, de las cuales el $57 \%$ son universidades, $26 \%$ centros de investigación y el restante $17 \%$ está representado en igual proporción entre empresas del estado y empresas privadas.

Del total de artículos con solo participación colombiana, en un $78 \%$ de estos hay la participación de una única institución colombiana y en el restante $22 \%$ de los artículos la relación interinstitucional predominante $(83 \%)$ está representada por alianzas universidad - centro de investigación o universidad - universidad; por tanto y como era de esperarse la relación universidad - empresa tiene una bajísima participación (6,6\%). Lo anterior denota, de una parte una escasa articulación entre los grupos colombianos y por otra la bajísima articulación con empresas privadas; lo cual se debe reflejar en la dispersión de temas abordados sin una aparente visión integral y estratégica de las acciones y de la predominancia de trabajos con una baja potencialidad de aplicación en sectores productivos (aspectos que quedarán evidentes cuando se analice más adelante los temas abordados por los grupos colombianos).

- En el restante 57\% de los artículos hay la participación de al menos otro país; en los cuales el tipo de articulación más común es en dúo (Colombia y otro país), representando el 83\% del total de artículos con cooperación internacional; los artículos producidos en trío de países (Colombia y dos países) representan un 14\% del total, destacándose que en $59 \%$ de éstos artículos la alianza es Colombia - país de América - país de Europa; 31\% Colombia países Europa; 7\% Colombia - países de América y 3\% Colombia - país de Asia - país de Europa; el 3\% restante del total de artículos tienen participación de más de tres países, interacción que está representada en un $60 \%$ por la relación Colombia - países de América - países de Europa, 20\% por Colombia - países de Europa y 20\% Colombia - países de América. Es importante tener en cuenta que la cooperación de Colombia con países de América se intensifica cuando hay de por medio un país europeo, lo cual fue corroborado en las entrevistas a los líderes ya que la alianza con el país americano amplia el espectro del problema a solucionar y la participación del país europeo fortalece tanto el componente tecnológico como las posibilidades de obtención de financiación.

Con base en la medición de Salton (Tabla II), la cooperación internacional presenta cuatro niveles de países claramente diferenciados. En el primero y con gran ventaja sobre los otros, España es el país con más intensa colaboración con Colombia; en el segundo nivel están dos países europeos (Francia y Alemania), Estados Unidos y Japón; en el tercer nivel están países americanos como Venezuela, Argentina, Chile y Brasil; y en el cuarto nivel están los demás países. Esta tendencia de un mayor incremento de cooperación internacional de Colombia con países europeos con relación a Estados Unidos también fue observada para el periodo 1986-1990 para algunas áreas biológicas (Narvaez-Berthelemot, et al, 1992). Con relación a la cooperación entre países latinoamericanos (exceptuando España), la baja colaboración entre estos ha sido una constante durante hace varios años (Lewison et al., 1993; Velho, 2001; Roa-Atkinson y Velho, 2005), principalmente debido a que a pesar de que comparten problemáticas comunes, las comunidades de investigación son pequeñas y los sistemas nacionales de CyT no son fuentes financiadoras importantes, por lo cual acuden a colaboraciones con países de mayor desarrollo.

- Cuando hay cooperación internacional, se sigue observando la misma tendencia mencionada anteriormente sobre la individualidad de participación de las instituciones colombianas, puesto que en el $80 \%$ de estos artículos hay presencia de una sola institución colombiana y en el $20 \%$ restante la relación interinstitucional predominante (94\%) es la misma mencionada anteriormente, corroborando la predominancia de la relación universidad universidad o centro de investigación y una bajísima interacción con grupos o autores de empresas.

- Cuando la relación de cooperación internacional es en dúo, el autor colombiano se posiciona como primer autor en $70 \%$ de los artículos $(86 \%$ con país 
de América, 64\% con país de Europa y 60\% con Japón); lo cual teniendo en cuenta la dinámica de orden de coautoría expresada por los líderes de los grupos colombianos en las entrevistas, indica que la relación de cooperación internacional se origina en gran parte desde Colombia hacia los otros países. Mientras que cuando el artículo es producido en trío o superior, el autor colombiano solamente se posiciona como primer autor en 31\% de los artículos (29\% cuando la relación es Colombia - país de América - país de Europa; 22\% Colombia - países de Europa; 50\% Colombia - países de América). Indicando una tendencia general de que cuando la participación es en trío o superior, Colombia es un país invitado a participar, mas no es este país quien propicia la participación.

- El principal nexo de cooperación internacional se origina especialmente con aquellos países en los cuales la persona líder del grupo ha realizado estudios de doctorado; es así, como del total de los líderes de grupos colombianos que trabajan en bioprospección que han realizado estudios de doctorado en el exterior, $66 \%$ los han llevado a cabo en España, Estados Unidos, Francia y Alemania; que son al mismo tiempo los países que participan en el $68 \%$ de los artículos mencionados.

- De acuerdo a la afiliación institucional expresada en los artículos, se observa que la relación con los 24 países (Tabla II) se realiza a través de 113 instituciones; las cuales en su mayoría son universidades y centros de investigación $(91 \%$ de la relación interinstitucional), con una bajísima interacción con empresas u otro tipo de institución académica o científica. Esta mecánica de relación interinstitucional se podría explicar por el hecho de que en Colombia las acciones de bioprospección son realizadas principalmente por grupos pertenecientes a universidades y centros de investigación con escasa participación de empresas privadas; quienes centran su accionar en actividades relacionadas con fases primarias de la práctica bioprospectiva, como estudios de caracterización, aislamiento, identificación, búsqueda, valoración y evaluación de compuestos, las cuales al no presentar una aplicación específica dificultan alianzas con empresas; esta evidencia se comprueba por las temáticas de publicación de estos grupos, lo cual se explicitará más adelante.

- omo se mencionó en la introducción de este trabajo, la mecánica de cooperación internacional puede ser influenciada por una gran variedad de factores; uno de los detectados en este estudio es la intensidad y laboriosidad de la persona líder del grupo. Es el caso específico de la relación con Japón (10\% de los artículos), cuyo nexo se debe principalmente a que la persona líder de un grupo, además de realizar estudios de doctorado en dicho país, posee una alta productividad en publicaciones y ha contribuido a la formación académica de jóvenes investigadores que han podido realizar intercambios con dicho país, cuyo principal resultado son publicaciones internacionales. Además, estos investigadores jóvenes una vez obtienen formación académica a nivel de doctorado crean y lideran sus propios grupos de investigación, manteniendo relación con el país cooperante. Otro de los factores que tiene influencia sobre el proceso de cooperación es la cercanía entre los países; es así como la cooperación con Venezuela ( $7 \%$ de los artículos) proviene casi de manera exclusiva de la relación de un grupo de una universidad colombiana situada cerca de la frontera con dicho país que incorpora estudiantes venezolanos en sus programas de posgrado.

\section{Temas abordados}

Los grupos colombianos que desarrollan actividades de investigación en bioprospección trabajan y publican sobre una gran gama de temas (tabla 3).

Los temas desarrollados por los grupos colombianos son los siguientes:

Principios bioactivos en vegetales: los trabajos están dirigidos hacia fases primarias del proceso bioprospectivo como la identificación y caracterización de principios que se podrían considerar bioactivos; para lo cual se exploran especies vegetales como las Annonaceas (10\% de 
TABLA 3

Principales temas y países cooperantes en temas relacionados con bioprospección

\begin{tabular}{|c|c|c|c|c|}
\hline Tema & $\begin{array}{l}N^{\circ} \\
\text { Art. }\end{array}$ & $\begin{array}{c}\% \\
\text { C.I. }\end{array}$ & Objeto principal & $\begin{array}{c}\text { Principales } \\
\text { Países } \\
\text { cooperantes }\end{array}$ \\
\hline $\begin{array}{l}\text { Principios } \\
\text { bioactivos en } \\
\text { vegetales. }\end{array}$ & 120 & 68 & $\begin{array}{l}\text { Identificación y caracterización de } \\
\text { principios con posible actividad } \\
\text { alcaloide o antiparasitaria. }\end{array}$ & $\begin{array}{l}\text { España; } \\
\text { Alemania; } \\
\text { Francia; } \\
\text { Japón. }\end{array}$ \\
\hline Bacterias. & 58 & 47 & $\begin{array}{l}\text { Identificación y caracterización de } \\
\text { Bacillus thuringensis y modelos de } \\
\text { crecimiento. }\end{array}$ & $\begin{array}{l}\text { E. Unidos; } \\
\text { España; } \\
\text { Alemania; } \\
\text { Inglaterra; } \\
\text { Francia. }\end{array}$ \\
\hline $\begin{array}{l}\text { Principios } \\
\text { bioactivos en } \\
\text { organismos } \\
\text { marinos. }\end{array}$ & 24 & 71 & $\begin{array}{l}\text { Identificación y caracterización de } \\
\text { principios obtenidos de esponjas y } \\
\text { corales con posible actividad citotóxica, } \\
\text { antifouling y bactericida. }\end{array}$ & $\begin{array}{l}\text { Japón; } \\
\text { España; } \\
\text { E. Unidos. }\end{array}$ \\
\hline $\begin{array}{l}\text { Sustancias } \\
\text { volátiles y aceites } \\
\text { esenciales en } \\
\text { vegetales. }\end{array}$ & 30 & 47 & $\begin{array}{l}\text { Aislamiento de sustancias volátiles y } \\
\text { aceites esenciales de frutas y arbustos. }\end{array}$ & Alemania. \\
\hline $\begin{array}{l}\text { Síntesis química } \\
\text { compuestos. }\end{array}$ & 35 & 77 & $\begin{array}{l}\text { Síntesis de sustancias con posible } \\
\text { aplicación antiparasitaria y aceites } \\
\text { esenciales. }\end{array}$ & $\begin{array}{l}\text { Venezuela; } \\
\text { España. }\end{array}$ \\
\hline $\begin{array}{l}\text { Enfermedades } \\
\text { parasitarias. }\end{array}$ & 15 & 53 & $\begin{array}{l}\text { Mecanismo de control para enfermedad } \\
\text { de Chagas y Leishmaniasis. }\end{array}$ & $\begin{array}{l}\text { España; } \\
\text { Chile; } \\
\text { Brasil. }\end{array}$ \\
\hline $\begin{array}{l}\text { Extracción de } \\
\text { compuestos. }\end{array}$ & 12 & 25 & $\begin{array}{l}\text { Métodos químicos para extracción de } \\
\text { sustancias volátiles. }\end{array}$ & $\begin{array}{l}\text { Venezuela; } \\
\text { España. }\end{array}$ \\
\hline $\begin{array}{l}\text { Organismos } \\
\text { marinos. }\end{array}$ & 10 & 30 & $\begin{array}{l}\text { Taxonomía y caracterización de } \\
\text { esponjas y corales. }\end{array}$ & $\begin{array}{l}\text { Chile; } \\
\text { España. }\end{array}$ \\
\hline
\end{tabular}

$\mathrm{N}^{\prime}$ Art.= número de artículos; \%C.I.= porcentaje de artículos en cooperación internacional.

los artículos), Musa acuminata (9\%), y en menor proporción trabajos relacionados con los géneros Solanum, Pasifloraceae y Piperaceae. Aunque estos cinco géneros vegetales son los más representativos de trabajos en esta temática, constituyen solamente un porcentaje cercano al 30\% del total de artículos; lo que evidencia el alto número de especies vegetales involucradas en este tipo de investigaciones, incluso muchas de las especies son referenciadas de manera individual en un solo artículo.

Ci. Inf., Brasília, DF, v. 38, n. 3, p.96-110, set./dez., 2009
Lo anterior demuestra una dispersión extraordinaria de trabajos que exploran una gran variedad de especies vegetales, sin que se observe una estrategia sistemática e integradora mediante la cual se expresen prioridades, focos comunes y trabajo articulado entre grupos. Esta tendencia de dispersión se corrobora al analizar el posible foco de dichos trabajos representado por una gran variedad de posibilidades como la identificación y caracterización de sustancias con actividad alcaloide (la cual presenta la mayor frecuencia, que no supera 
el 9\% de los artículos), actividad antiparasitaria (especialmente contra leishmaniasis), actividad antiinflamatoria, actividad antioxidante, producción de fitoalexinas, actividad citotóxica, actividad antifúngica y actividad antitumoral.

Un 68\% del total de artículos de esta temática presentan relaciones de cooperación internacional, destacándose alianzas con países como España, Francia, Alemania y Japón; y en un segundo plano países como Bolivia, Argentina, Brasil y Chile. Estadística que corrobora lo mencionado anteriormente, en cuanto a que la mecánica de cooperación internacional tiene una fuerte relación con el país en el cual los líderes de los grupos realizaron estudios de posgrado y que la relación con países americanos se produce en un segundo plano, principalmente motivadas por la necesidad de compartir algunas problemáticas comunes.

Las entrevistas realizadas con líderes de grupos de esta temática corroboran la existencia de una serie de experiencias de cooperación internacional enfocadas a lo académico e investigativo; representadas en la participación de redes como CYTED y ALFA Europea; en publicaciones conjuntas con universidades y centros de investigación; formación académica de estudiantes colombianos de posgrado en varias universidades de distintos países y organización y participación de cursos internacionales en productos naturales realizados en varios países.

Bacterias: $60 \%$ de los trabajos de este tema se pueden considerar que abordan aspectos básicos para identificación de bacterias, modelos de crecimiento y producción de endotoxinas; el 40\% restante de los artículos denota alguna posibilidad de aplicación industrial de dichas bacterias. Es así, como un $82 \%$ de estos estudian y analizan a Bacillus thuringensis $(\mathrm{Bt})$ en su actividad antimicrobial, mosquicida e insecticida y como controladora de plagas y enfermedades agrícolas; el restante 18\% de los artículos examinan algunas cepas de bacterias en su efecto para bioremediación en pozos petroleros y capacidad microbial para producción de biopolímeros. Esta temática, comparada con la anteriormente analizada, guarda la semejanza de enfocarse principalmente sobre aspectos básicos relacionados con identificación y caracterización; pero se diferencia en que en la parte aplicada hay un centro de atracción representado por la bacteria Bt, la cual puede ser considerada como la bacteria más estudiada a nivel mundial.

Del total de estos trabajos, 53\% son publicaciones realizadas por grupos colombianos sin participación internacional, indicando que los grupos colombianos que trabajan en esta área temática han fortalecido tanto capacidades básicas como aplicadas. La cooperación internacional se proporciona tanto para trabajos que abordan aspectos básicos (52\% de los artículos), principalmente en colaboración con Estados Unidos; como en trabajos que denotan alguna potencialidad de aplicabilidad ( $44 \%$ de los artículos), destacándose la participación de países europeos como España, Alemania, Inglaterra y Francia y una baja participación de países como Brasil, México, Argentina, Chile y Costa Rica.

A través de las entrevistas a los diferentes líderes de los grupos que trabajan en esta temática se pudo percibir y corroborar los siguientes aspectos:

- En el caso de Bt, la dinámica se ha impulsado bastante debido a la conformación de redes en las cuales hay participación de varios países, especialmente latinoamericanos como Colombia, Costa Rica, México, Argentina, España y Chile; y otros países como Brasil e Inglaterra; redes cuyo principal objetivo es la caracterización de las colecciones locales de cepas de Bt. para determinar su potencial uso en agricultura y salud pública. En el caso específico de Colombia, el principal resultado de participación en este tipo de red es la caracterización y determinación del potencial insecticida de aproximadamente 100 cepas de Bt., la capacitación del recurso humano mediante pasantías cortas para entrenamiento de estudiantes de posgrado, publicaciones nacionales e internacionales, compra de equipos de laboratorio por valores aproximados de USD \$ 40.000 y recepción de aportes en efectivo de USD \$ 200.000 para gastos de investigación.

Ci. Inf., Brasília, DF, v. 38, n. 3, p.96-110, set./dez., 2009 
- Con relación a trabajos de bioremediación, estos se centran en articulación universidad con industrias multinacionales petroleras para lo cual se realizan actividades en proteómica y genómica mediante las cuales se identifican, caracterizan, evalúan, seleccionan y multiplican microorganismos que posteriormente se devuelven a su ambiente para que hagan el trabajo de remediación en sitios en los cuales ya se hizo la exploración. El beneficio conjunto de esta articulación consiste en que el secreto industrial de la actividad de bioprospección es propiedad de la empresa; mientras el beneficio para la universidad consiste en pagos del costo de personal del grupo de investigación que pueden representar hasta un 65\% del total de estos.

- En cuanto a capacidad microbial para producción de biopolímeros (levanas), la investigación que se ha adelantado permite contar con un biopolímero de origen microbiano obtenido mediante la selección de microorganismos nativos. En la actualidad la ejecución de este tipo de trabajos ha obtenido una patente para proceso y producto y se está desarrollando la etapa de escalamiento mediante una alianza universidad - empresa colombiana, en la cual la empresa tiene la exclusividad de la explotación potencial del biopolímero para uso farmacéutico (recubrimiento de medicamentos). En esta alianza la universidad ha invertido una cifra aproximada de USD\$ 170.000 para los trámites de patente en Japón, Estados Unidos y Europa y la empresa ha realizado por un lapso de 7 años para el desarrollo y escalamiento del biopolímero inversiones cercanas a los USD \$ 1,5 Millones. Por la patente obtenida, la universidad ha sido demandada por el Ministerio de Ambiente de Colombia, argumentándose el patentamiento de un producto para el cual no se contaba con el permiso respectivo para acceder a recursos genéticos (para mayor información sobre la normatividad legal consultar: Casas, 1999; Comunidad Andina, Normativa Andina, On Line; FAO, On Line; Ministerio del Medio Ambiente, Vivienda y Desarrollo Territorial de Colombia, On line; Duarte y Velho, 2008).
- Continuando con la aplicabilidad de resultados a nivel industrial con el uso de bacterias y microorganismos, otra alianza universidad - empresa, ha generado cuatro productos para el cultivo de arroz que ya cuentan con permiso de comercialización: 2 fijadores de Nitrógeno, 1 biocontrolador para plagas y 1 solubilizador de Fósforo. Los agricultores han invertido durante un período de 8 años una suma aproximada de USD \$ 1,0 Millón; creándose una spin-off llamada Biocultivos S.A., a través de la cual se han desarrollado los cuatro productos mencionados, los cuales se han probado en 15.000 ha de arroz. Actualmente, se está evaluando la posibilidad de patentar los productos y procesos y el acuerdo con la universidad es que a ésta le correspondería el 2,5\% sobre ventas en el mercado.

- Algunos grupos colombianos vienen trabajando en bioprospección y mapeo de microorganismos en ambientes extremos en parques naturales de Colombia para lo cual están usando técnicas de metagenómica y bioinformática con el principal objetivo de conocer la diversidad microbiana con potencial en la industria farmacéutica y cosmética. En esta iniciativa participan 4 universidades colombianas en alianza con la Universidad de Harvard (departamento de genética) y un instituto de Brasil para desarrollos en bioinformática. Los trabajos previamente realizados por los grupos colombianos demuestran la enorme biodiversidad existente en esos ambientes, para lo cual ya se han realizado los respectivos aislamientos encontrando nuevas especies y nuevos géneros de microorganismos. Con base en estos resultados se ha establecido contacto con empresas francesas de base tecnológica que compran esa biodiversidad, las cuales exigen la entrega de todos los microorganismos del interés de ellos y solamente reconocerían algún incentivo económico después de la venta posiblemente en dos años, cuando consigan clientes a quien vendérselos, Con experiencias como esta se corrobora que tanto los investigadores como las instituciones colombianas no poseen ninguna capacidad para negociar con las empresas, requiriéndose la contratación de profesionales expertos en negociación tecnológica. Además, estas experiencias denotan otro inconveniente 
relacionado con las publicaciones internacionales ya que las cepas objeto de publicación quedan de libre acceso a nivel mundial; ante lo cual, los líderes de los grupos colombianos opinan que se debe seguir la estrategia de no realizar publicaciones sobre las cepas de mayor potencial económico y comercial. Es así, como los líderes de los grupos reconocen que a través de la cooperación internacional los mayores beneficios siguen teniendo relación con actividades de fortalecimiento académico e investigativo como la formación de estudiantes, publicaciones, pasantías al exterior de los investigadores y mejora en la infraestructura de laboratorios de investigación.

Principios bioactivos de organismos marinos: estos trabajos realizan investigaciones principalmente en identificación y caracterización de esponjas y corales evaluando potencial de actividad citotóxica, antifouling, bactericida y producción de alcaloides. Un 71\% de los artículos se desarrollan conjuntamente con países como Japón (30\%) España (25\%), Estados Unidos (8\%) y otros países como Italia, Francia y Brasil.

Además de estos trabajos hay 10 artículos que investigan la taxonomía y caracterización de organismos marinos; estudios que contribuyen para complementar los trabajos de productos bioactivos. En esta parte más básica, existe menor proporción de cooperación internacional (30\% de los artículos), mediante articulación con países como Chile, España y Puerto Rico.

Por el tipo de actividades desarrolladas por los grupos que trabajan en esta área temática; hay unos grupos que se dedican de manera exclusiva a lo que se consideraría como investigación básica desarrollando acciones relacionadas con la identificación y caracterización de estructuras secundarias de RNA, cuyos principales resultados son el acceso a colecciones biológicas, la capacitación de estudiantes en el exterior, el uso de equipos robustos y proyectos y publicaciones conjuntos. Otros grupos, aunque también trabajan en actividades básicas, han estado adquiriendo experiencias mediante alianzas con empresas.
Es así, como hay experiencias de grupos colombianos con empresas españolas como el Instituto Biomar y PharmaMar, alianzas mediante las cuales el componente académico e investigativo (responsabilidad de los grupos colombianos) tenía el compromiso de producir compuestos con potencial bioactividad y el compromiso de las industrias españolas era el de probar dicho potencial. Estas experiencias alcanzaron avances interesantes en la identificación y composición de la estructura química de los compuestos, pero no se logró la obtención de bioactividad de los compuestos, lo cual originó la desarticulación de la alianza por parte de las empresas. Estas experiencias son ejemplos concretos de las diferencias en ritmos e intereses entre lo académico-investigativo y lo comercial, diferencias que los grupos colombianos han entendido perfectamente y en la actualidad están preparándose para abordarlos de una forma diferente.

Los trabajos más recientes en este temática han originado el hallazgo de compuestos medicinales de alto potencial antiinflamatorio (llamados pseudoterosinas) provenientes de coral marino; trabajos realizados mediante alianzas académicas de grupos académicos colombianos con universidades como Stanford, California, Hawaii, La Coruña, Santa Catarina y Buenos Aires; y cuyos resultados están listos para integrarse a la industria, pero aún no hay alianzas concretas. Tal vez esta sea una excelente oportunidad para revivir las alianzas con las empresas españolas mencionadas anteriormente.

Se destaca en esta temática los trabajos desarrollados con 70 esponjas marinas, cuyos estudios químicos y evaluación de bioactividad han permitido la identificación y caracterización de 300 compuestos químicos, de los cuales ya se han aislado 70 estructuras novedosas con un enorme potencial de actividad antimicrobiana y antitumoral (Duque, 2009).

Una tendencia interesante observada en esta área temática, adoptada por algunos grupos colombianos, está relacionada con el enfoque reciente (desde hace unos 4 años) hacia trabajos en recubrimientos

Ci. Inf., Brasília, DF, v. 38, n. 3, p.96-110, set./dez., 2009 
antifouling, cuya potencialidad puede ser vista como una gran alternativa a las enormes dificultades que atraviesan los grupos de investigación de países en desarrollo por el alto costo de la investigación en compuestos para producir medicamentos; además, la otra ventaja que presenta el futuro desarrollo en recubrimientos antifouling, con relación al de medicamentos, es que en Colombia existe una industria nacional de pinturas, la cual podría utilizar los productos que se obtengan para ser usados en barcos y plataformas submarinas.

Es así, como algunos de los líderes de los grupos colombianos que desarrollan esta temática, mencionan que en la actualidad los trabajos y publicaciones ya tienen un enfoque más preciso sobre la potencialidad del compuesto estudiado y que por lo tanto están dispuestos a trabajar de manera más articulada con las empresas, con el fin de ir disminuyendo la desconfianza mutua actualmente existente y de esta forma lograr que la academia piense en productos que generen renta para las universidades y que las industrias entiendan los tiempos para los desarrollos científicos.

\section{Aislamiento de sustancias volátiles de vegetales y aceites esenciales vegetales: el énfasis de esta temática se relaciona con trabajos en los cuales se realiza la caracterización química de sustancias pertenecientes a frutos y otros vegetales. Es así, como 75\% del total de estos artículos demuestran trabajos en una diversidad de frutas, como mora (Rubus glaucus), granadilla (Passiffora edulis), uchuva (Physalis peruvian), melón (Cucumis melo), papaya (Carica papaya), tamarillo (Cyphomandra betacea), lulo (Solanum quitoense), curuba (Passiflora cumbalensis), badea (Passiflora cumbalensis), guayaba (Psidium guajava) y mamey (Mammee apple); el restante 25\% en otros vegetales arbustivos como Ylang Ylang (Cananga odorata), Limpia origanoides; copaica (Copaifera officinalis) y escobillón rojo (Callistemon specious).}

En el caso de frutales, se observa que la tendencia generalizada es la generación de un artículo de investigación por cada frutal, en el cual se describe la estructura química de los principales compuestos volátiles. La finalidad de estos trabajos básicos es la identificación y aislamiento del compuesto volátil, el cual por medio de síntesis química permite la producción de sustancias volátiles con características similares a las naturales, las cuales tienen un buen potencial de incorporación en la industria alimenticia como aromatizantes o saborizantes.

$53 \%$ del total de estos artículos están publicados con la participación de solamente autores colombianos. El restante $47 \%$ a través de cooperación con países como Alemania (33\%) y entre 1-2 artículos en colaboración con países como Japón, España, Estados Unidos y Uruguay.

Además de los artículos mencionados anteriormente, hay 12 artículos en los cuales se describen métodos químicos para extracción de compuestos; de los cuales el $50 \%$ son específicos a sustancias volátiles y el restante 50\% para extracción de sustancias como gliceroles, esteroles y flavonoides. El 75\% de estos artículos son producidos por autores colombianos, denotando capacidad endógena en este tema.

En cuanto a experiencias relacionadas con extracción de aceites esenciales, en Colombia se viene desarrollando esta actividad en varios grupos de investigación, los cuales mediante una convocatoria pública obtuvieron recursos del gobierno colombiano por USD \$ 1,8 Millones para la conformación desde hace cuatro años de un centro de investigación de excelencia para la agroindustrialización de especies vegetales aromáticas y medicinales tropicales (CENIVAM, on line). Dicho Centro está conformado interdisciplinariamente por 9 grupos de investigación de 5 universidades colombianas, una fundación y 2 industrias privadas colombianas (Stachenko, 2009). Durante estos cuatro años el Centro ha enfocado sus actividades en las etapas de recolección de material vegetal (colectándose más de 350 accesiones de vegetales); análisis cromatográfico de volátiles; transformación catalítica (81 sustancias nuevas sintetizadas); ensayos de bioactividad (antibacterial, antimicótica, toxicidad aguda y antilehismania), trabajo en plantas piloto y transferencia de tecnologías. La experiencia de este Centro ha sido bastante interesante ya que ha permitido articular el trabajo interdisciplinario de varios grupos y alianzas 
entre la academia y el sector productivo; además, ha permitido avanzar en experiencias mediante las cuales se fijan prioridades y el gobierno colombiano apoya con recursos financieros de importante cuantía.

Con relación a trabajos relacionados con sustancias volátiles, en la actualidad en Colombia existe un par de casos empresariales exitosos que han permitido introducir al mercado nacional aromas artificiales en golosinas, producidos a través de síntesis química de los aromas naturales; lo cual se logró mediante una alianza de 5 años entre la universidad y la industria colombiana.

Síntesis de compuestos químicos: son 35 artículos los que reportan procedimientos y metodologías para síntesis química de compuestos con potencial aplicación contra enfermedades parasitarias (principalmente leishmaniasis), actividad antifúngica y citotóxica, utilización de aceites esenciales, contra efectos sicóticos y producción de ácidos carboxílicos. $23 \%$ de estos artículos han sido generados por instituciones y autores colombianos y en el restante hay colaboración internacional, especialmente con Venezuela (37\% de los artículos con cooperación internacional), España (37\%) y Francia, Inglaterra y Argentina (cada uno con 7\%).

Enfermedades parasitarias: 15 artículos mencionan trabajos específicos para control de enfermedades como Chagas (80\% de los artículos) y Leishmaniasis (20\%); en estos trabajos se identifican y caracterizan clones y cepas de parásitos del género Trypanosoma y se estudia el control de vectores de los agentes parasitarios. $47 \%$ de estos artículos son generados por grupos colombianos y en el restante hay cooperación internacional, especialmente de España (37\% de los artículos con cooperación internacional), Chile (37\%) y Brasil (25\%). Esta es el área temática en la cual se presenta mayor articulación entre países del Sur, debido a que comparten problemas similares. La articulación de los grupos colombianos con grupos de países del Norte se relaciona desde el punto de vista académico (especialmente a través de estudiantes de posgrado) y no se detectó alianzas con empresas.
Otros temas: el restante de artículos tratan sobre una gama de temas relacionados por ejemplo con identificación y caracterización de hongos (5 artículos), variabilidad genética de vegetales (4), transgénesis en plantas (3), productos bioactivos en hongos (3), control biológico de plagas y enfermedades agrícolas (3); química básica (3), identificación y caracterización de insectos que son plagas agrícolas (2), vacunas genéticas (2), y otros temas como biocatálisis, impacto ambiental marino, fisiología de frutales amazónicos; entre otros.

\section{CONCLUSIONES}

Los grupos colombianos tienen la tendencia general de publicar sin generar colaboraciones de otras instituciones nacionales, lo cual reflejaría que realizan trabajos de manera independiente, originando investigaciones en una gran cantidad de temas. Tendencia que debe ser corregida a través de acciones de política de CyT como la que ha venido propiciando este país desde hace unos cuatro años a través del apoyo por parte de recursos públicos a consorcios de grupos de investigación para que emprendan trabajos de manera coordinada y conjunta, por medio de estrategias como la que se ha denominado conformación de Centros de Investigación de Excelencia.

Para el tema de bioprospección, existe mayor articulación de los grupos colombianos con grupos de otros países que con grupos nacionales. Observándose que las principales motivaciones de esta articulación por parte de los investigadores colombianos son preferencialmente de carácter académico e investigativo, y solo durante los últimos años se observa alguna preocupación de realizar trabajos con enfoques más aplicados y selectivos, cuyos resultados se puedan incorporar y negociar con empresas.

España es el país con el cual Colombia mantiene los mayores niveles de cooperación, seguido por otros países europeos como Francia, Alemania e Inglaterra y posteriormente los Estados Unidos. Se observó que el grado de cooperación con los

Ci. Inf., Brasília, DF, v. 38, n. 3, p.96-110, set./dez., 2009 
países guarda una estrecha relación con el país en el cual el líder del grupo colombiano haya realizado sus estudios de posgrado. También, se observó que un polo importante de cooperación se relaciona con las posibilidades de obtener financiación cuando se participa en redes temáticas, lo cual se detectó especialmente para redes europeas. También, pareciera existir una pequeña tendencia de diferenciación de la articulación de cooperación con países europeos o con los Estados Unidos; ya que con los primeros se muestra una mayor potencialidad de realizar trabajos con aplicabilidad en sectores empresariales; mientras que con los Estados Unidos la principal y más importante motivación es de tipo académico.

La cooperación entre Colombia y países americanos (exceptuando Estados Unidos) se presenta en un tercer nivel, motivada principalmente por la oportunidad de compartir problemas comunes relacionados con condiciones tropicales.

Por el análisis efectuado en este trabajo, creemos que en la actualidad la temática de bioactividad de organismos marinos es la que presenta más avances en bioprospección en Colombia, tanto desde el punto de vista básico como aplicado. Destacándose la presencia desde hace varios años de grupos que han venido trabajando de manera muy intensa, con lo cual han contribuido al relacionamiento con grupos internacionales, a la presencia de un gran número de publicaciones internacionales, a la formación académica de investigadores y a la formación de otros grupos jóvenes en Colombia.

Colombia ha venido sentando las bases para la conformación de un Programa Estratégico en Bioprospección, necesitándose de medidas específicas de política de CyT como la identificación de prioridades y mayor apoyo a grupos de investigación que trabajen coordinadamente en las áreas prioritarias identificadas.

Ci. Inf., Brasília, DF, v. 38, n. 3, p.96-110, set./dez., 2009

\section{AGRADECIMIENTOS}

- A los 12 líderes colombianos de grupos de investigación quienes compartieron a través de entrevista sus experiencias: Carmenza Duque, Dolly Montoya y Alejandro Chaparro, de la Universidad Nacional de Colombia; Sergio Orduz, Centro de Investigaciones Biológicas; Patricia del Portillo, CORPOGEN; Juan Armando Sánchez, Jenny Dussán, Mauricio Linares, Silvia Restrepo, de la Universidad de Los Andes; Sandra Baena, Rubén Torrenegra, de la Universidad Javeriana; Fernando Echeverri, de la Universidad de Antioquia.

- A Coordenação de Aperfeiçoamento de Pessoal de Nível Superior (CAPES, Brasil), por el apoyo a través de una bolsa de estudios.

Artigo submetido em 09/06/2009 e aceito em 27/01/2010.

\section{REFERÊNCIAS}

ARTUSO, A. (2002). Bioprospecting, Benefit Sharing, and Biotechnological Capacity Building. World Development, Vol. 30, No. 8, pp. 1355-1368.

BOISVERT, V.; CARON, A. (2002). The Convention on Biological Diversity: An Institutionalist Perspective of the Debates. Journal of Economic Issues. Vol. XXXVI No. I, March, pp 151-166.

BRAND, U.; GÖRG, C. (2003). The State and the Regulation of Biodiversity, International Biopolitics and the Case of Mexico. Geoforum, Vol. 34 (2): 221-233.

BRUSH, S. (1999). Bioprospecting the Public Domain. Cultural Anthropology. Vol 14 (4): 535-55.

CABRERA, J. (2000). El Acceso a los Recursos Genéticos y los Sistemas Sui Generis como Mecanismo para la Protección del Conocimiento Tradicional: La Experiencia Costarricense. UNCTAD Expert Meeting on Systems and National Experiences for Protecting Traditional Knowledge, Innovations and Practices. Geneva. 30 October-1 November 2000. Consultada Novembro de 2006. http:// r0.unctad.org/trade_env/tk.htm

CALERO, C.; VAN LEEUWEN, T.N.; TIJSSEN, R.J.W. (2007). Research cooperation within the bio-pharmaceutical industry: Network analyses of co-publications within and between firms. Scientometrics, 71 (1): 87-99

CASAS A. (1999). Recursos genéticos, biodiversidad y derecho. Bogotá. Ediciones jurídicas Gustavo Ibañez e Instituto Colombiano de Derecho Ambiental. 25 p.

CASTREE, N. (2003). Bioprospecting: from theory to practice (and back again). Transactions of the Institute of British Geographers, 28, 1: 35-55.

CENIVAM. Centro de Investigación de Excelencia para la Agroindustrialización de Especies Vegetales Aromáticas y Medicinales 
Tropicales (On line). Consultada en Enero de 2009. Disponible en http://cenivam.uis.edu.co/principal.php

COMUNIDAD ANDINA, NORMATIVA ANDINA. (On line). Consultada Julio de 2008. Disponible en URL:http://www. comunidadandina.org/normativa/dec/D391.htm.

COLCIENCIAS, (2008). Colombia Construye y Siembra Futuro. Política Nacional de Fomento a la Investigación y la Innovación. Instituto Colombiano para el Desarrollo de la Ciencia y la Tecnología - COLCIENCIAS -. $67 \mathrm{p}$.

DUARTE, O.; VELHO, L. (2008). Análisis del marco legal en Colombia para la implementación de prácticas de bioprospección. Acta Biológica Colombiana. 13(2): 103-122.

DUQUE, C. (2009). Organismos marinos: fuente asombrosa de compuestos útiles para la humanidad. En: Carlos Corredor; Felipe Guhl; Carmenza Duque (editores) Memorias Seminario Internacional Tendencias y Futuro de la Investigación en Parasitología y en Productos Naturales. ACOFACIEN y ACCEFYN. Editora Guadalupe S.A, Bogotá, Colombia, páginas 353 - 372.

ESCOBAR, A. (1999). After Nature: Steps to an Antiessentialist Political Ecology. Current Anthropology, 40 (10): 1-30.

FAO. Organización de las Naciones Unidas para la Agricultura y la Alimentación (On line). Convenio sobre la Diversidad Biológica. Consultada Julio de 2008. http://www.fao.org/biodiversity/ CBD_es.asp

FEINSILVER, J.M., (1996), Prospección de la biodiversidad: potencialidades para los países en desarrollo". Revista de la CEPAL n. 60, Diciembre, pp111-128.

GEORGHIOU, L. (1998). Global cooperation in research. Research Policy. Vol. 27, pages 611- 626.

GLANZEL, W.; SCHUBERT, A.; CZERWON, H.-J. (1999). A bibliometric analysis of international scientific cooperation of the European Union (1985-1995). Scientometrics, 45 (2): 185-202

HENNE, G. and FAKIR, S. (1999). NBI-Ball Agreement: A new phase in bioprospecting?. Biotechnology and Development Monitor, No. 39, pages $18-21$

INBIO, INSTITUTO NACIONAL DE BIODIVERSIDAD DE COSTA RICA. (On line) Consultada septiembre 2008. Disponible en http://www.inbio.ac.cr.

KATSOUYANNI, K. (2008). Collaborative research: Accomplishments \& potential. Environ Health. 7:3. Published online 2008 January 21. http://www.pubmedcentral.nih.gov/articlerender. fcgi? artid $=2265690$

KATZ, J. S., MARTIN, B. R. (1997): What is research collaboration? Research Policy, 26:1-18.

LAIRD, S. A. (2002) Introduction: equitable partnerships in practice. In: Biodiversity and Traditional Knowledge. Equitable Partnerships in Practice. Edited By Sarah A. Laird. Earthscan Publications Ltd, London, Sterling, VA. pages xxii-xxxvi.

LAIRD, S; ten KATE, K. (2002). Biodiversity prospecting: the commercial use of genetic resources and best practice in benefit-sharing. In: Biodiversity and Traditional Knowledge. Equitable Partnerships in Practice. Edited By Sarah A. Laird. Earthscan Publications Ltd, London, Sterling, VA. Section IV, Chapter 8. pages 241-286
LAIRD, S. A.; WYNBERG, R. (2002). Institutional policies for biodiversity research. In: Biodiversity and Traditional Knowledge. Equitable Partnerships in Practice. Edited By Sarah A. Laird. Earthscan Publications Ltd, London, Sterling, VA. Section I, Chapter 3 , pages 39-76.

LEWISON, G.; FAWCETT-JONES, A.; KESSLER, C. (1993). Latin-American scientific outputs 1986-1991 and international coauthorship patterns, Scientometrics, Vol. 27 (3): 317-336.

MELGAREJO, L. M., J. SÁNCHEZ, A. CHAPARRO, F. NEWMARK, M. SANTOS-ACEVEDO, C. BURBANO y C. REYES. (2002). Aproximación al estado actual de la bioprospección en Colombia Bogotá: Cargraphics, 334p. Serie de Documentos Generales INVEMAR No.10)

MINISTERIO DEL MEDIO AMBIENTE, VIVIENDA Y DESARROLLO TERRITORIAL DE COLOMBIA. (On line). Consultada diciembre de 2008. Disponible en URL: http: $\backslash \backslash w w w$. minambiente.gov.co

MORAN, K.; KING, S.; CARLSON, Th. (2001). Biodiversity Prospecting Lessons and Prospects Annual Review of Anthropology 30: 505-526.

NARVAEZ-BERTHELEMOT, N., FRIGOLETTO, L.P. and MIGUEL, J.F. (1992). International scientific collaboration in Latin America, Scientometrics, Vol. 24 (3): 373-392.

ROA-ATKINSON, A. (2004). Partnership in Bio prospecting in Colombia: North-South, Public-Private Partnerships in biotechnology relevant issues and impact in the developing countries. Final report UNU-INTECH.

ROA-ATKINSON, A.; VELHO, L. (2005). Interactions in knowledge production: A comparative case study of immunology research groups in Colombia and Brazil. Aslib Proceedings: New Information Perspectives, 57 (3): 200-216. www.emeral dinsight.com/researchregister/0001-253X.htm.

SCHMOCH, U.; SCHUBERT, T. (2008). Are international co-publications an indicator for quality of scientific research? Scientometrics, 74 (3): 361-377.

STACHENKO, E. (2009). Perspectivas de la agroindustria de aceites esenciales. En: Carlos Corredor; Felipe Guhl; Carmenza Duque (editores) Memorias Seminario Internacional Tendencias y Futuro de la Investigación en Parasitología y en Productos Naturales. ACOFACIEN y ACCEFYN. Editora Guadalupe S.A, Bogotá, Colombia, páginas 385-398.

SWIDERSKA, K. (2001). Stakeholder Participation in Policy on Access to Genetic Resources, Traditional Knowledge and BenefitSharing, Case Studies and Recommendations. Biodiversity and Livelihoods Issues No. 4. March 2001. Pages 1-35.

VELHO, L. (2001). Redes regionais de cooperação em C\&T e o Mercosul. (On line) http://ftp.unb.br/pub/download/ipr/rel/ parcerias/2001/2607.pdf

VELHO, L. (2004). North-South, Public-Private, Collaboration in Biotechnology: Relevant Issues and Impact in Developing Countries (COLBIOTECH). Consultada Julho de 2008. http://www.intech. unu.edu/research/currentresearch/global/velho/2000_162.php.

Ci. Inf., Brasília, DF, v. 38, n. 3, p.96-110, set./dez., 2009 